\title{
Environmental behavior and impacts of antibiotics
}

\author{
Yaozong CUI 1,2, a, Yanhong $\mathrm{LI}^{1,2, \mathrm{~b}}$, Lihao ZHANG ${ }^{1,2, \mathrm{c}}$ and Nan XIAO 1,2, d \\ ${ }^{1}$ Guangxi Key Laboratory of Environmental Pollution Control Theory and Technology, \\ Guilin University of Technology, Guilin 541004, China; \\ ${ }^{2}$ Collaborative Innovation Center for Water Pollution Control and Water Safety in Karst Area, \\ Guilin University of Technology, Guilin 541004, China
}

ayaozongcui16@163.com, blyh1685@163.com, 08zhanglihao@163.com, d597320102@qq.com

\begin{abstract}
Keywords: antibiotics; determination method; sorption and degradation; antibiotic resistance genes; toxicological effects

Abstract. Antibiotics are widely used to treat or prevent human and animal diseases, as well as to promote the growth of animals in livestock breeding and aquaculture. As a type of antibacterial drugs, antibiotics have been widely applied in human/animal disease prevention, disease treatment, animal husbandry and aquaculture, etc. A majority of antibiotics introduced into human/animal cannot be utilized directly, leading to the result that more than $85 \%$ antibiotics were discharged into the environment. Once antibiotics enter the ecosystems, they could influence the evolution of the community structure, which according affect the ecological function of aquatic environment. Correspondingly, antibiotic resistant bacteria (ARB) have been found, which is threatening ecological safety and human health. In this review, five parts were discussed in detail: (1) the source of antibiotics in the environment; (2) the determination of antibiotics and antibiotic resistance genes (ARGs) in the environment; (3) the spatial distribution of antibiotics and in the environment; (4) the fate of antibiotics in the environment; (5) the toxicological effects of antibiotics. Moreover, the existing problems and future research directions are proposed.
\end{abstract}

\section{Introduction}

Antibiotics are defined as drugs of natural, semisynthetic or synthetic origin with antibacterial, antifungal or antiparasitic activity. Until now, antibiotics have been applied in treating and preventing human and animal diseases as one of the most effective drug treatments. However, antibiotics could also induce ARB, becoming the important environmental pollutants. Large amounts of antibiotics introduced into human/animal cannot be utilized directly, leading to the result that more than $85 \%$ antibiotics were discharged into the environment in the form of treated or untreated wastewater [1].

However, the inappropriate use of antibiotics has caused serious environmental pollution recently. In pathogenicity and environmental microbiology, the proliferation and development of antibiotic resistance have been a public health problem [2]. Antibiotics with the characteristics of persistent and difficult biodegradability have the potential harm to human, land and sea creatures. More severely, the use of antibiotics could induce the development of antibiotic resistant bacteria and ARGs. Feces are used as fertilizer from animal feeding could also be a potential source of environmental antibiotic contamination [3].

\section{The Use of Antibiotics}

Generally speaking, the antibiotics are mainly used in both human and veterinary medicine. With the development of living standards, people will pay more attention to their own health. At present, antibiotics are also abused in hospital for pharmaceutical purpose.

For a long time, the abuse of antibiotics in China has been one of the most serious environmental problems in the world. In developed countries in Europe and the United States, the use of antibiotics could reach to about $10 \%$ of all drugs, while the hospitals in China accounted for at least $30 \%$. 
In addition, the use of antibiotics can effectively treat the infections and prevent the health of animals. In the process of poultry production, the reasonable use of antibiotics could promote the growth of poultry quickly. It was reported that the United States had supplied about 11,200 tons of veterinary antibiotics in production, such as pigs, cattle or others. Besides, the similar situation was also occurred in Africa, Europe, New Zealand and other countries [4].

\section{Sources and Pathways for Antibiotic Residues in the Environment}

Antibiotics in the environment have many sources which are animal husbandry, factory emissions, pharmaceuticals, aquaculture, etc. After consumption, antibiotics are usually excreted and are metabolized only partially via feces or urine (figure 1 ).

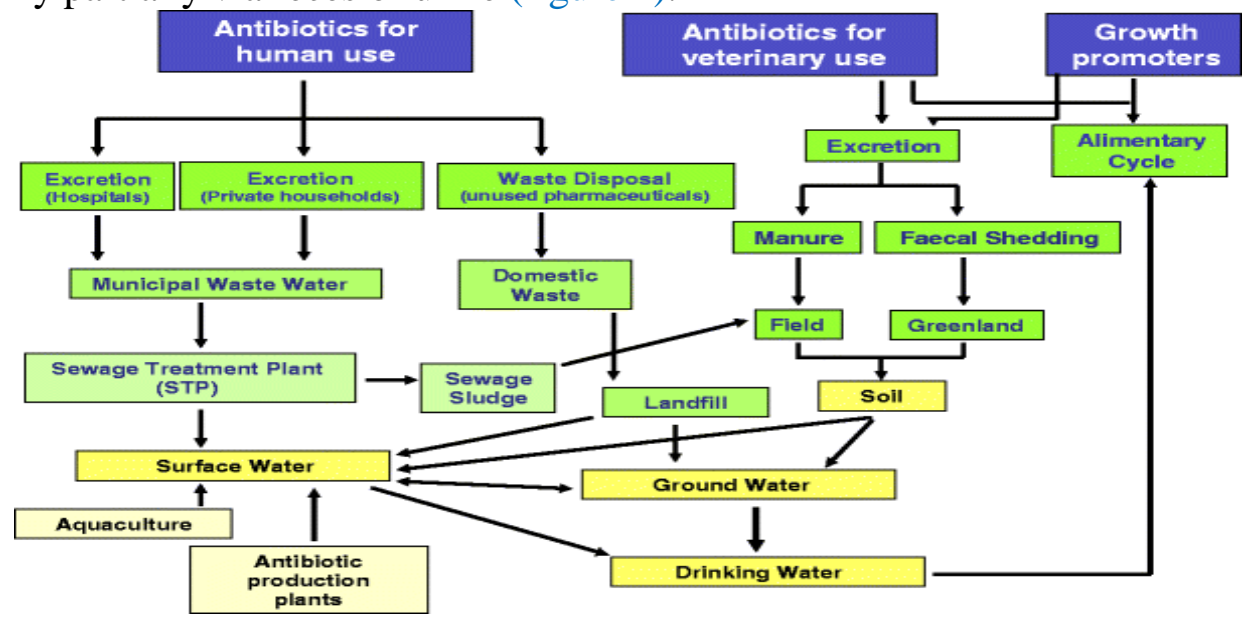

Fig.1 Sources and pathways for antibiotic residues in the environment ${ }^{[5]}$

There are about $80 \%$ often partially metabolized in organisms and excreted as the primary compounds or metabolites into the environment via feces and urine. After consumption, antibiotics are discharged into the urban sewage treatment plants (STPs), which cannot be effectively removed from environment [6]. Once antibiotics enter in the environment, the inactive drug, and including metabolites, they could be transported and degraded. Effluents from the STPs are released into surface waters and ground waters, which may cause the pollution of drinking water.

Antibiotics have attracted a great deal of attention during the last decade, especially in the field of animal husbandry and aquaculture. Extensive use of veterinary antibiotics in livestock breeding increases risk that these compounds ends up in the environment when metabolites are used as fertilizer. There are some overdue without formal handling of antibiotic drugs directly into the trash, besides, pharmaceutical wastewater directly discharged into the aquatic environment, which could cause pollution.

\section{Detection Methods}

At present, some different standard methods have been developed to determine the concentration of antibiotics in the aquatic environment, activated sludge, and sediments samples. Due to the polar nature, thermal stability and low volatility of these antibiotics, they need derivatization before detection. Thus, liquid chromatography (LC) has become the technique of solution for multiclass methods, particularly when coupled to mass spectrometry (MS) and tandem MS (LC-MS-MS), the LC-MS ${ }^{2}$ allowing improved selectivity and sensitivity in much more complex matrices [7]. Despite these characteristics, due to the relatively low antibiotic concentration levels predicted in most food and water samples, the main detection need a preconcentration step, for sample extraction proceduces, before analysis. The final target analytes are detected by LC-MS-MS. Solid phase extraction is usually performed in offline mode, while online provides a choice for samples. Future trendencies in the application of LC-MS-based techniques to multiclass antibiotic methods are also proposed. 


\section{The Current Status of Antibiotics Contamination}

Because of the large application amount of antibiotics and their wide distribution, they can be detected in STPs, soils, sediments, and biological samples, and even in drinking water. Among them, the detection of high frequency of antibiotics has macrolides, quinolones, sulfonamides, tetracycline.

Current Situation of Antibiotics Pollution in Water Environment. At present, the STPs, hospital, aquaculture and pharmaceutical manufactures wastewater have been detected in the existence of a majority of antibiotics with the concentration from $\mu \mathrm{g} \cdot \mathrm{L}^{-1}$ to $\mathrm{mg} \cdot \mathrm{L}^{-1}$. In 2009 , Hojosa et al.[8] found that the influent concentration was $46.0 \mu \mathrm{g} \cdot \mathrm{L}^{-1}$ and the effluent concentration was 30.0 $\mu \mathrm{g} \bullet \mathrm{L}^{-1}$ for amoxicillin in STPs in Spain. In 2006, Watkinson et al. [9] reported that the ciprofloxacin (CIP) concentration in Australia of detection for influent concentration was $3.80 \mu \mathrm{g} \cdot \mathrm{L}^{-1}$ and the effluent concentration was $0.64 \mu \mathrm{g} \cdot \mathrm{L}^{-1}$, respectively.

Pollution Status of Antibiotics in Sludge and Sediments. Part of the antibiotic entering the STPS enters the surface water with the effluent, and the other part also enters into the activated sludge during the treatment process. In addition, a large number of the antibiotics in the surface water will transport into the sediments, leading to the accumulation effect of antibiotics which cannot be degraded in sediments and activated sludge. In 2008, Zhou et al. [10] found that the concentrations of the NOR and oxytetracycline in Liaohe basin in China ranged from not detection to $120 \mu \mathrm{g} \cdot \mathrm{kg}^{-1}$ and from not detection to $76.6 \mu \mathrm{g} \cdot \mathrm{kg}^{-1}$, respectively. In 2004, Lindberg et al. [11] found that the concentrations of the CIP in activated sludge samples in Sweden ranged from 5500 to $6300 \mu \mathrm{g} \cdot \mathrm{kg}^{-1}$.

\section{The Degradation of Antibiotics in the Environment}

The degradation of antibiotics in the environment could be classified as biodegradation and abiotic degradation. Non-biodegradation includes chemical degradation, photodegradation and hydrolysis.

Antibiotics Removal in STPs. As far as our knowledge, the STPs which become the point source of enriched antibiotics cannot completely remove all antibiotics. In a study by Gan et al. [12] investigations were conducted in sludge and sewage from a STP in southwestern China regarding the behavior and fate of typical antibiotics. Results showed that biotransformation and degradation were the main removal mechanisms. For quinolone antibiotics and azithromycin, sludge sorption was also a removal way (sludge sorption accounted for 9.35\% 26.96\% of the influent water load).

Degradation of Pollutants in Water. Hydrolysis is one of the important ways of antibiotic degradation. For example, $\beta$-lactams have been found in the environment at very low concentrations and they are easily hydrolyzed. Boreen et al. [13] found that photodegradation rate for sulfonamide antibiotics was very slow.

\section{Environmental Effect of Antibiotics}

Problem of antibiotic resistance genes (ARGs). The use of antibiotics could lead to the emergence of ARB or ARGs, which contain the potential environment risks to humans. The environmental persistence, migration and transformation of ARGs are more detrimental than antibiotics themselves, which have attracted ever growing attention as a newly emerging environmental issue.

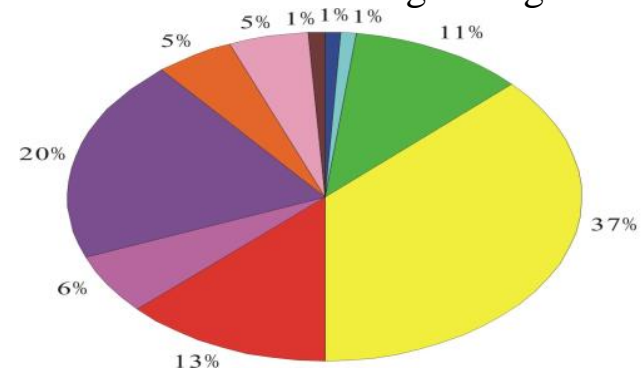

口 Tetracycline ARGs in soil

- Sulfanilamide ARGs in soil

- Chloramphenicol ARGs in soil

- Aminoglycoside ARGs in soil

- Tetracycline ARGs in activated sludge

- Sulfanilamide ARGs in activated sludge

- Tetracycline ARGs in sediment

- Sulfanilamide ARGs in sediment

- Quinolone ARGs in sediment

- Others

Fig.2 Research distribution for different ARGs in the soil environment of China ${ }^{[14]}$ 
In Figure 2, the studies about ARGs research in China from 2009 to 2014 were reviewed, and the distribution of ARGs such as soil, activated sludge and sediments were included. Analysis of soil environment showed tetracycline ARGs in soil to be in highest percentage (at least $37 \%$ ), followed by tetracycline ARGs in activated sludge.

It was found that the content of ARGs in sediments was higher than that in surface water. Zhou et al. [3] reported that the concentration of ARGs in sediments was 120 2000 times higher than that in the aquatic environment in the Haihe River Basin in Tianjin. Stoll et al. [15] detected 24 resistance genes from a river basin in Europe, proving sulfonamides and streptomycin ARGs at $77 \sim 100 \%$ and $43 \sim 55 \%$, respectively.

Ecological Toxicity of Antibiotics. The ecological toxicity of antibiotics and their metabolites in the environment are mainly as follows: (1) inhibit of plant and animal growth and development; (2) influence the community structure and ecological function, thereby affect soil fertility; (3) induce antibiotic resistant bacteria, and lead to an increasing concern about the potential environmental and public health risk.

Antibiotics on the Toxicity of Plants. Toxicity of antibiotics on plant size is dependent on the kinds of antibiotics and plants, also on the antibiotic use and soil sorption ability and so on.

Environment medium could significantly affect the toxicity of antibiotics. In 1982, Batchelder et al.[16] found that tetracycline had little effect on bean yield in the clay soil, but bean production decreased in the sandy soil.

Toxicity of Antibiotics to Animals. Previous publications have shown that tetracyclines, sulfonamides and quinolones and other antibiotics have strong soil sorption, leading to a long environment retention time. Some types of antibiotics (tylosin and oxytetracycline) showed low toxicity. Wollenberger et al. [17] reported that some of the antibiotics used in aquaculture can inhibit growth for insects and aquatic organisms, and even kill them. The studies have shown that antibiotics on aquatic invertebrates or fish had moderate toxicity. $\mathrm{EC}_{50}$ values after 48 hours was found in the range of $25 \mathrm{mg} \cdot \mathrm{L}^{-1}$ to $500 \mathrm{mg} \cdot \mathrm{L}^{-1}$ with Daphnia magna as the most potent.

\section{Research and Prospects of Antibiotics}

It is of great practical and theoretical significance to study the environmental behavior and ecotoxicology of antibiotics; and to establish the safety assessment and early warning system for the ecological environment of antibiotics; to protect human health and the ecological environment. In addition, we should analyze the ARGs pollution and environmental behavior with risk evaluation.

\section{Acknowledgements}

This work was financially supported by the Guangxi Scientific Experiment Center of Mining, Metallurgy and Environment (KH2012ZD004) and the project of high level innovation team and outstanding scholar in Guangxi colleges and universities (002401013001).

\section{References}

[1] A. Hartmann, A.C. Alder and T. Koller: Identification of fluoroquinnolone antibiotics as the main source of human genotoxicity in native hospital water[J]. Environmental Toxicology and Chemistry. Vol. 17(1998), p.377

[2] B. Halling-Sorensen, S. Nors Nielsen and P.F. Lanzky: Occurrence, Fate and Effects of Pharmaceutical Substances in the Environment[J]. Chemosphere. Vol. 36(1998), p.357

[3] Y. Luo, D.Q. Mao and Q.X. Zhou: Trends in Antibiotic Resistance Genes Occurrence in the Haihe River, China[J]. Environmental Science and Technology. Vol. 44( 2010), p.7720 
[4] S.C. Kim and K. Carlson: Temporal and spatial trend in the occurrence of human and veterinary antibiotics in aqueous and river sediment matrices[J].Environmental Science and Technology. Vol. 41(2007), p.50

[5] M.C. Moreno-Bondi, M. Dolores Marazuela and S. Herranz: an overview of sample preparation procedures for LC-MS multiclass antibiotic determination in environmental and food samples[J]. Analytical and Bioanalytical Chemistry. Vol. 395(2009), p.921

[6] W.H. Xu, G. Zhang, S.C. Zou and X.D. Li: Determination of selected antibiotics in the Victoria Harbour and the Pearl River, South China using high-performance liquid chromatography electrospray ionization tandem mass spectrometry[J]. Environmental Pollution. Vol. 145(2007), p.672

[7] M.C. Moreno-Bondi, M. Dolores Marazuela and S. Herranz: an overview of sample preparation procedures for LC-MS multiclass antibiotic determination in environmental and food samples[J]. Analytical and Bioanalytical Chemistry. Vol. 395(2009), p.921

[8] M. Hijosa-Valsero, G. Fink and M.P. Schlusener: Removal of antibiotics from urban wastewater by constructed wetland optimization[J]. Chemosphere. Vol. 83(2011), p.713

[9] A.J. Watkinson, E.J. Murby and S.D. Costanzo: Removal of antibiotics in conventional and advanced wastewater treatment: implications for environmental discharge and wastewater recycling[J]. Water Research. Vol. 41(2007), p.4164

[10]L.J. Zhou, G.G. Ying and J.L. Zhao: Trends in the occurrence of human and veterinary antibiotics in the sediments of the Yellow River, Hai River and Liao River in northern China[J]. Environmental Pollution. Vol. 159(2011), p.1877

[11]R.H. Lindberg, U. Olofsson, and P. Rendahl: Behavior of Fluoroquinolones and Trimethoprim during Mechanical, Chemical, and Active Sludge Treatment of Sewage Water and Digestion of Sludge[J]. Environmental Science and Technology. Vol. 40(2006), p.1042

[12]Xiumei. Gan, Qing. Yan and $\mathrm{Xu}$. Gao: Occurrence and fate of typical antibiotics in wastewater treatment plant in southwest China[J]. Environment Science. Vol. 35(2014), p.1817 (In Chinese)

[13]A.L. Boreen, W.A. Arnold and K. Mcneill: Photochemical Fate of Sulfa Drugs in the Aquatic Environment: Sulfa Drugs Containing Five Membered Heterocyclic Groups[J]. Environmental Science and Technology. Vol. 38(2004), p.3933

[14] Yiwen. Shen, Zhiting. Huang and Bing. Xie: Advances in research of pollution, degradation and removal of antibiotics and antibiotics resistance genes in the environment[J]. Chinese Journal of Applied and Environmental Biology. Vol. 21(2015), p.181 ( In Chinese )

[15]C. Stoll, J.P. Sidhu and A. Tiehm: Prevalence of clinically relevant antibiotic resistance genes in surface water samples collected from Germany and Australia[J]. Environmental Science and Technology. Vol. 46(2012), p. 9716

[16]A.R. Batchelder: Chlortetracycline and oxytetracycline effects on plant growth and development in solid system[J]. Journal of Environment Quality. Vol. 11(1982), p.675

[17]L. Wollenberger, B. Halling-Sorensen and K.O. Kusk: Acute and chronic toxicity of veterinary antibiotics to Daphnia magna[J]. Chemosphere. Vol. 40(2000), p.723 